\title{
Special issue on quality management for information systems
}

\section{Mario Piattini $^{1}$ • Ignacio García-Rodríguez de Guzmán ${ }^{1}$ • Ricardo Pérez-Castillo ${ }^{1}$}

Published online: 21 April 2020

C) Springer Science+Business Media, LLC, part of Springer Nature 2020

\section{Introduction to the special issue}

From the industry point of view, IS (Information Systems) Quality is much more than achieving adequate levels of quality in the systems of which company IS is composed. IS quality has become a strategic objective that directly impacts the success of all companies.

However, since technologies and paradigms (both those supporting software and those that develop it) are in continuous evolution, quality methods and tools must also be updated.

It is also crucial to (i) identify what the current trends and the most influential technologies are (such as big data and machine learning), and (ii) reconsider traditional processes (for example, software design, development and testing), aiming to reformulate mechanisms, and in doing so ensure quality and maintain the value these processes bring to business.

This special issue is composed of 11 papers which present a realistic point of view of current research and practice, and provide the state of the art regarding the quality of IS engineering and maintenance.

The first two papers focus their proposals on Service Quality. In "Using the IDEAL model for the Construction of a Deployment Framework of IT Service Desks at the Brazilian Federal Institutes of Education", Cristiano Domingues da Silva and Alexandre Marcos Lins de Vasconcelos focus on the lack of IT support services in the Brazilian FIs (Federal Institutes of Education, Science and Technology). The paper proposes (i) a deployment/improvement approach, based on the IDEAL model, using practices from models such as ITIL, ISO 20000,

This article is part of the Topical Collection on Quality Management for Information Systems Guest Editor: Mario Piattini, Ignacio García Rodríguez de Guzmán, Ricardo Pérez del Castillo

\section{Ricardo Pérez-Castillo}

Ricardo.PDelCastillo@uclm.es

Mario Piattini

Mario.Piattini@uclm.es

Ignacio García-Rodríguez de Guzmán

Ignacio.GRodriguez@uclm.es

1 Institute of Technologies and Information Systems, University of Castilla-La Mancha, Paseo de Moledores, s/n, 13071 Ciudad Real, Spain 
CMMI-SVC and MR-MPS-SV; and (ii) a Process Toolbox that follows the seven-dimension structure of EPMF. In the second proposal entitled "Data-driven and Tool-supported Elicitation of Quality Requirements in Agile Companies", Marc Oriol, Silverio Martínez-Fernández, Woubshet Behutiye, Carles Farré, Rafal Kozik, Pertti Seppänen, Anna Maria Vollmer, Pilar Rodríguez, Xavier Franch, Sanja Aaramaa, Antonin Abhervé, Michal Choras and Jari Partanen present an interesting industrial data-driven elicitation process to identify quality requirements from software development repositories in agile software developments. A Automated support enables practitioners to identify violations of existing quality-related issues, which is incorporated in the product backlog.

Under the topic of Software Security, two papers have been included. In "Assessing Data Cybersecurity Using ISO/IEC 25012”, Javier Verdugo and Moisés Rodríguez present a framework to evaluate the degree of data cybersecurity. The approach proposes (i) a quality model, (ii) an evaluation process and (iii) a tool to support the visualization of the results of the assessment. The second paper entitled "Integrating Security and Privacy in Software Development", by Maria Teresa Baldassarre, Vita Santa Barletta, Danilo Caivano and Michele Scalera, presents the POSD (Privacy Oriented Software Development) approach, based on the five key elements: Privacy by Design, Privacy Design, Strategies, Privacy Pattern, and Vulnerabilities and Context. POSD is intended to discover software vulnerabilities either in software development (forwards, to produce a secured system) or over legacy systems (backwards, to reengineer into a secured version).

Regarding Data Quality, in "Towards a software quality certification of Master Data-based Applications", Fernando Gualo, Ismael Caballero and Moisés Rodríguez introduce a framework to certify that master-data based applications meet the stated requirements identified in ISO 8000 parts 100-140, by (i) following an approach based on the certification of software product quality from ISO 25010 and (ii) starting from a set of functional requirements the authors extracted from the aforementioned parts of ISO 8000. Continuing with Data Quality, Julio Souza, Diana Pimenta, Ismael Caballero and Alberto Freitas define in "Measuring data credibility and medical coding: a case study using a nationwide portuguese inpatient database" a method based on artificial intelligence techniques to measure credibility in APR-DRG databases at hospitals. Such databases are prone to be corrupted (with untrustworthy information). The automated identification of such information improves the database credibility.

On focusing on Software Quality, two papers were selected. In "Code Smell Detection using Multi-label Classification Approach", Thirupathi Guggulothu and Salman Abdul Moiz present a machine learning approach to identify multiple code smells affecting the same code element. In "Specification and Use of Concern Metrics for supporting Modularity-Oriented Modernizations", Daniel San Martin, Guisella Angulo, Bruno Santos, Raphael Honda and Valter Vieira Camargo deal with the problem of poorly modularized concerns, which have an impact on the maintenance of legacy systems. The ADM-based approach uses the SMM (Structured Metrics Metamodel, from OMG) to measure the level of concern modularization in a legacy system to perform modularity-oriented modernizations to improve software quality.

Finally, three papers deal with the Software Testing concern, which is actually a process in continuous evolution. This being the case of "Two Experiments for Evaluating the Impact of Hamcrest and AssertJ on Assertions Development", where Maurizio Leotta, Maura Cerioli, Dario Olianas and Filippo Ricca focus on the emerging automation techniques that enhance the productivity of testers, in the endeavour to support the resource demands in continuous testing, agile methods and DevOps. Two assertion frameworks from different approaches are compared and assessed under the criteria of correctness and time required to develop the 
assertions. The approach put forward in "RETORCH: An Approach for Resource-aware Orchestration of End-to-End Test Cases" proposed by Cristian Augusto Alonso, Jesús Morán Barbón, Antonia Bertolino, Claudio de la Riva and Javier Tuya deals with End-to-End testing. This situation presents an important drawback; whole system availability is required for execution of test cases (now emphasized in DevOps). This proposal improves that situation by means of (i) an optimization based on a resource-aware test orchestration and (ii) scheduling of the execution of the test cases in several machines according to the optimization. Finally, Software Testing considers the scenario of the absence of models representing a web application. In "Test Case Generation Based on Mutations Over User Execution Traces”, Ana Paiva, André Restivo and Sérgio Almeida propose a web testing approach that generates test cases from execution traces and applies mutation to enrich the test suite. This approach enables testers to test software systems where there may be a complete lack of knowledge of software structure.

Acknowledgement As the guest editors, we thank all the authors for their valuable contributions, the referees who reviewed the papers in this issue and from the Software Quality Journal. We thank Rachel Harrison (Editorin-Chief) and Katherine Moretti (Assistant Editor) for their continuous support.

Publisher's note Springer Nature remains neutral with regard to jurisdictional claims in published maps and institutional affiliations.

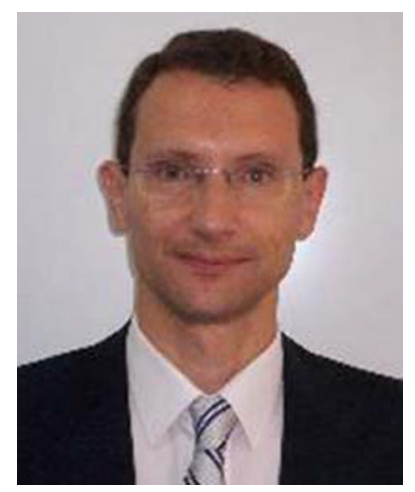

Mario Piattini MSc. (1989) and Ph.D. (1994) in Computer Sciencefrom Madrid Technical University (UPM). PMP, CISA, CISM, CGEITand CRISC. Founder-Director of the Information Systems andTechnologies of the UCLM (University of Castilla-La Mancha) and ofthe UCLM-INDRA Research and Development Joint Center.Between the "15 Top scholars in the field of systems and software engineering(2004-2008)" and the "Among the 15 "Most active experienced SE researchers(2010-2017)". Full Professor of Software Engineering at UCLM, and leader of theAlarcos Research Group and scientific director of AQCLab, S.L. (first ENAC / ILACaccredited laboratory for software and data quality based on ISO 25000). Currentlyleader of the aQuantum scientific research team (Alarcos Group). 


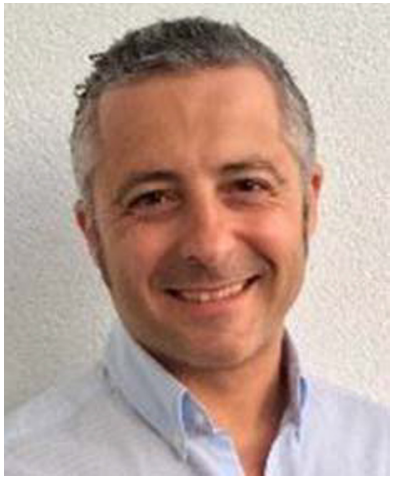

Ignacio García Rodríguez de Guzmán obtained the degrees ofTechnical Engineer in Computer Systems (2001), ComputerEngineer (2003) and Doctor in Computer Engineering (2007) at theUniversity of Castilla-La Mancha. He has participated as acollaborator in several research projects. He is co-author of morethan 85 publications. In addition, he has participated as a member of the organizingcommittee and program committee of several conferences. He has obtained theprofessional certifications of CISA (Certified Information System Auditor), ScrumManager and ISTQB for software testing. He enjoys the position of Director of theITSI (Institute of Technologies and Information Systems) since 2015. Currentlymember of the aQuantum scientific research team (Alarcos Group) where he workson quantum software model-driven modernization.

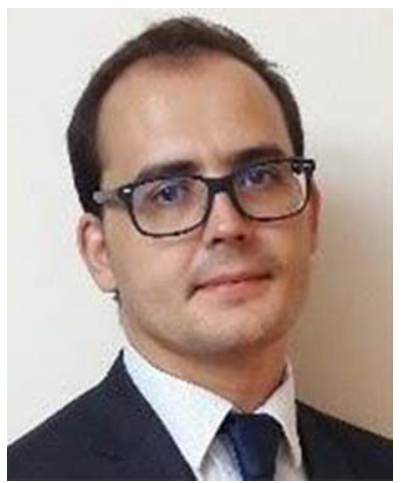

Ricardo Pérez-Castillo holds the Ph.D. degree in Computer Sciencefrom the University of Castilla-La Mancha (Spain). He works at theIT \& Social Sciences School of Talavera at University of Castilla-LaMancha. His research interests include architecture-drivenmodernization, model-driven development and business processarchaeology. Currently member of the aQuantum scientific research team where heworks on the migration of classical systems to quantum architectures and quantumsoftware reengineering. 